\title{
Fortschreibung des Status Quo - Die EU und ihr neuer Finanzrahmen Agenda 2007
}

\author{
Peter Becker*
}

Inzwischen wiederholen sich alle sechs Jahre die schwierigen und äußerst konfliktreichen Verhandlungen über den mehrjährigen Finanzrahmen der Europäischen Union. Und wie so oft wurden auch unmittelbar nach dem Kompromiss der Staats- und Regierungschefs Mitte Dezember 2005 erneut die kritischen Stimmen lauter, die eine grundsätzliche Reform der europäischen Finanzverfassung forderten. ${ }^{1}$

Wie in einem Brennglas konzentrieren sich in diesen Verhandlungen über die Eckpunkte der mittelfristigen EU-Finanzierungs- und Ausgabenplanung alle internen Verteilungskonflikte. Alle EU-Organe, die Mitgliedstaaten, die über das Eigenmittelsystem maßgeblich den Haushalt der EU finanzieren, sowie die Vielzahl der europäischen Interessen- und Lobbygruppen, die von Ausgabenkürzungen oder -steigerungen in einem Politikbereich betroffen sein könnten, haben naturgemäß ein großes Interesse daran, ihre eigenen Prioritäten in diese Verhandlungen einzubringen und durchzusetzen.

Allerdings sind bislang weder die Finanzielle Vorausschau selbst noch das förmliche Verhandlungsverfahren im europäischen Primärrecht verankert, das heißt es besteht weder eine formelle Verpflichtung, diese mittelfristige Finanzplanung vorzulegen, noch gibt es einen vorgegebenen Verhandlungsrahmen. Dennoch hat sich auf der Grundlage der bisherigen drei Verhandlungsrunden zu den Finanzpaketen Delors I, Delors II und zur Agenda 2000 ein ,politischer acquis‘ entwickelt, der auch den Rahmen für die Agenda 2007-Verhandlungen bildete. Dieser Haushaltsacquis manifestiert sich sowohl in den Verhandlungsthemen, den besonderen Konfliktlinien zwischen den antagonistischen Interessen der beteiligten Akteure als auch in den einzelnen Verfahrensschritten sowie dem besonderen Ablauf der Verhandlungen.

Die Verhandlungen über die Finanzielle Vorausschau sind insoweit mehrdimensional: die politisch-materielle Dimension bezieht sich auf die künftige Schwerpunktsetzung europäischer Politik, die fiskalische auf die Verteilung der Finanzierungslasten zwischen den Mitgliedstaaten und schließlich die verhandlungstaktische Dimension auf das bargaining zwischen den Mitgliedstaaten. Dieser Dreiklang bildet den Hintergrund zur Analyse des bisherigen Verhandlungsprozesses zur Agenda 2007. Zuvor soll jedoch eine erste politische Bewertung des Gipfelergebnisses vom Dezember 2005 vorgenommen und die Eckpunkte der Einigung im Europäischen Rat nachgezeichnet werden.

\section{Die politische Gipfelbilanz}

Die Erleichterung war allenthalben groß als es den europäischen Staats- und Regierungschefs nach mehr als 30 Stunden intensiver Verhandlungen in der Nacht vom 16. zum 17. Dezember 2005 endlich gelungen war, einen Kompromiss über den mehrjährigen Finanzrahmen der erweiterten Europäischen Union für die Jahre 2007 bis 2013 zu vereinbaren. Diese überaus schwierigen Verhandlungen waren nach dem Scheitern im Juni 2005 und den nega-

* Peter Becker, Forschungsgruppe EU-Integration, Stiftung Wissenschaft und Politik, Berlin.

1 Vgl. „Barroso fordert nach Streit um EU-Haushalt ,völlig neues Verfahren““, in: Handelsblatt vom 20.12.2005. 
tiven Verfassungsreferenden in Frankreich und den Niederlanden zu einem weiteren Symbol der Zerrissenheit und der Krise in der erweiterten Union geworden. ${ }^{2}$

Dennoch hat die Europäische Union mit dieser politischen Einigung bestenfalls ihre Handlungsfähigkeit bewiesen; eine grundsätzliche und umfassende Modernisierung der europäischen Finanzverfassung und des EU-Haushalts, wie sie Tony Blair noch in seiner fulminanten Rede vor dem Europäischen Parlament am 23. Juni 2005 zu Beginn der britischen Präsidentschaft gefordert hatte, konnte mit diesem Kompromiss nicht erreicht werden. Das Ergebnis vom Dezember schreibt vielmehr den strukturkonservativen europäischen Status quo fort - sowohl die Prioritäten auf der Ausgabenseite als auch die fortdauernde Intransparenz und Komplexität des Eigenmittelsystems folgen den eingefahrenen Bahnen.

Um aber überhaupt zu einer Einigung zu gelangen, bedurfte es einer zugespitzten Verhandlungsdynamik und einer besonderen Krisensituation, damit die ,Drohung des Scheiterns" ausreichenden Entscheidungs- und Konsensdruck erzeugen konnte. Durch ihre ,Hinhaltetaktik' verstärkte die britische Präsidentschaft noch den Druck. Während sie im Oktober 2005 noch zu einem lediglich informellen Gipfeltreffen ohne Beschlussvorlagen nach Hampton Court einlud und die Agenda 2007-Themen nicht ansprechen wollte drängten alle anderen EU-Partner London dazu, einen eigenen Vorschlag vorzulegen, um die Verhandlungen fortzusetzen. Trotz der auch über die Medien lancierten Unzufriedenheit in nahezu allen Hauptstädten hielt der Vorsitz an seinem Zeitplan fest und legte erst am 5. Dezember einen eigenen Kompromisstext vor. Jedoch sollte auch dieser Vorschlag offensichtlich weniger als Grundlage für einen ausgewogenen Kompromiss, sondern als Menetekel dienen, welche Verhandlungsposition London einnehmen würde, wenn eine Einigung im Dezember scheitern würde und London dann nicht mehr zu einer vermittelnden Rolle als Vorsitz gezwungen wäre. Die späte Vorstellung eines neuen Vorschlags nur zehn Tage vor dem entscheidenden Gipfel erhöhte zudem den Einigungsdruck auf die Nettoempfänger. Bei einem nochmaligen Scheitern der Verhandlungen liefen sie Gefahr, dass die EU-Programme, von denen sie zu profitieren hofften, verspätet anlaufen würden. Dies würde nicht nur zu einer verkürzten Förderperiode, sondern in der Konsequenz auch zu einem verringerten Mittelvolumen führen.

London hatte mit seinem ersten Entwurf zugleich auch die Verhandlungsspielräume neu abgesteckt. Mit der nochmaligen deutlichen Kürzung des luxemburgischen Vorschlags wurde die von den fünf Nettozahler geforderte 1-Prozent-Zielmarge endgültig zur Verhandlungsgrundlage der letzten Runde. Der Vorschlag der EU-Kommission vom Februar 2004, mit dem die eigentliche Verhandlungsgrundlage formuliert worden war, spielte keine Rolle mehr. Die Verhandlungsmarge war auf die Differenz zwischen dem letzten luxemburgischen Papier vom 17. Juni und dem zweiten britischen Vorschlag vom 14. Dezember 2005, also rund 133,7 Millionen Euro zusammen geschmolzen (Schaubild 1). Kompensationen und kleinere Korrekturen wurden vornehmlich über die Liste der Sonderregelungen und technisch-administrative Anpassungen gesucht.

2 Vgl. z.B. „Die EU leidet an Lähmungserscheinungen“, in: Neue Zürcher Zeitung vom 20.06.2005; Michael Stabenow: „Traum und Trauma“, in: Frankfurter Allgemeine Zeitung vom 20.06.2005. 


\section{Schaubild 1: Gegenüberstellung der Vorschläge für den Finanzrahmen 2007-2013 (in Mrd. Euro)}

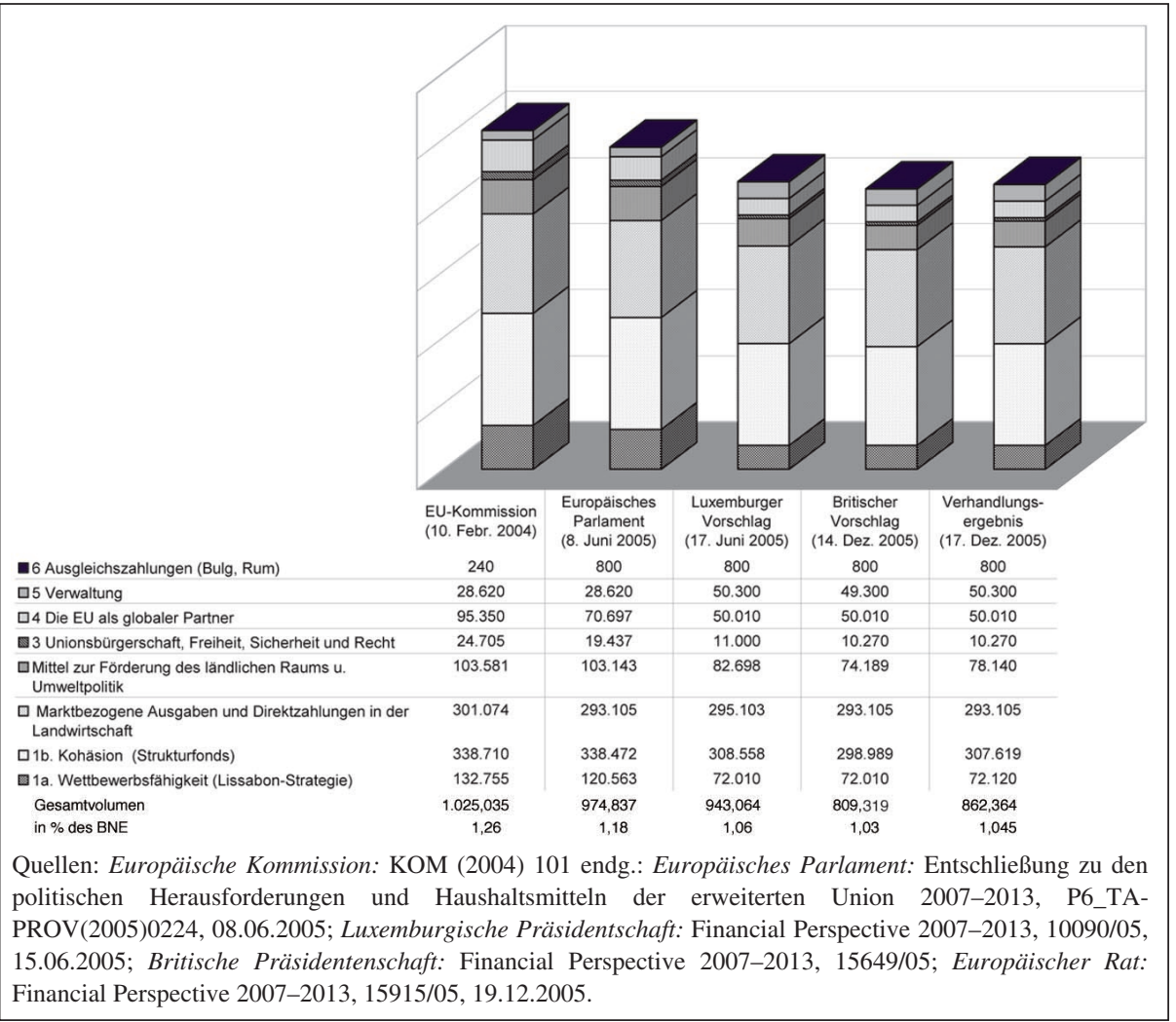

Über allem stand aber wie bereits im Juni die Notwendigkeit, ausreichende finanzielle Manövriermasse auf der Einnahmenseite zu erzeugen, um die nationalen Sonderzahlungen finanzieren zu können. Die Bereitschaft Tony Blairs, auf einen größeren Teil der Zuwachses des britischen Beitragsrabatts zu verzichten, war hierfür ebenso notwendig wie die deutsche Bereitschaft, durch die Aufstockung des Gesamtvolumens die deutschen Abführungen an den EU-Haushalt zu erhöhen, und das französische Zugeständnis, auf eine Anpassung des Agrarkompromisses an die Aufnahme Bulgariens und Rumäniens zu verzichten.

Gerade der deutschen Gipfeldiplomatie wuchs vor dem Hintergrund der restriktiven britischen Verhandlungsstrategie und der ebenso starren französischen Position (nicht mehr am Agrarkompromiss vom Oktober 2002 zu rütteln) fast zwangsläufig die Rolle des fairen Maklers zu. Unmittelbar nach dem Gipfel wurde diese Verhandlungsstrategie der neuen deutschen Bundesregierung und insbesondere die neue Bundeskanzlerin Angela Merkel gelobt. ${ }^{3}$ Auch wenn die Rolle Deutschlands und der Kanzlerin in späteren Kommentaren wie-

3 Vgl. „Außenansicht: Viel Lob für Angela Merkel“ in: Handelsblatt vom 19.12.2005; „Der Merkel-Gipfel“ in: Süddeutsche Zeitung vom 17. 12. 2005; „Sie kam, sah - und gefiel“, in: Frankfurter Allgemeine Sonntagszeitung vom 18.12.2005. 
der relativiert wurde ${ }^{4}$ bleibt festzuhalten, dass die deutsche Gipfeldiplomatie den eigenen Handlungsspielraum in der EU erweitert hat. Neben die sichtbare und am Verhandlungsergebnis ablesbare enge Zusammenarbeit mit Frankreich trat die Annäherung sowohl an Großbritannien wie auch an die kleineren EU-Mitgliedstaaten.

\section{Die Eckpunkte des neuen Finanzrahmens}

Die Staats- und Regierungschefs verständigten sich für den Siebenjahreszeitraum des Finanzrahmens auf ein Gesamtvolumen von 862,363 Milliarden Euro oder 1,045 Prozent des EU-Bruttonationaleinkommens (BNE). ${ }^{5}$ Den ersten Vorschlag der Europäischen Kommission vom Februar 2004 über ein Gesamtvolumen von 1025,035 Milliarden Euro hat der Europäische Rat damit um über 162 Milliarden zusammengestrichen. In Relation zum erwarteten Wirtschaftswachstum der EU sinkt der Anteil des EU-Haushalts leicht von 1,09 Prozent im Jahr 2007 auf 1,0 Prozent des EU-BNE im Jahr 2013; das heißt, die Wachstumsraten der europäischen Wirtschaft werden höher sein als die Wachstumsraten des EU-Haushalts.

Auch weiterhin bleiben 70 Prozent der Haushaltsmittel für die Gemeinsame Agrarpolitik und die europäische Kohäsionspolitik gebunden. Von den insgesamt rund 862 Milliarden Euro sollen rund 307 Milliarden für die europäischen Strukturfonds und rund 293 Milliarden für die marktbezogenen Agrarausgaben und die Direktzahlungen an die Landwirte ausgegeben werden. Allerdings sinkt der Anteil der marktbezogenen Ausgaben der Gemeinsamen Agrarpolitik langsam im Verlauf der sieben Jahre, während die Haushaltsplafonds der Strukturfonds stetig anwachsen; bereits im Jahr 2008 sollen die Ausgaben für die Strukturfonds die marktbezogenen Ausgaben und die Direktzahlungen im Bereich der Landwirtschaft übertreffen (Schaubild 2).

\section{Schaubild 2: EU-Finanzrahmen 2007-2013}

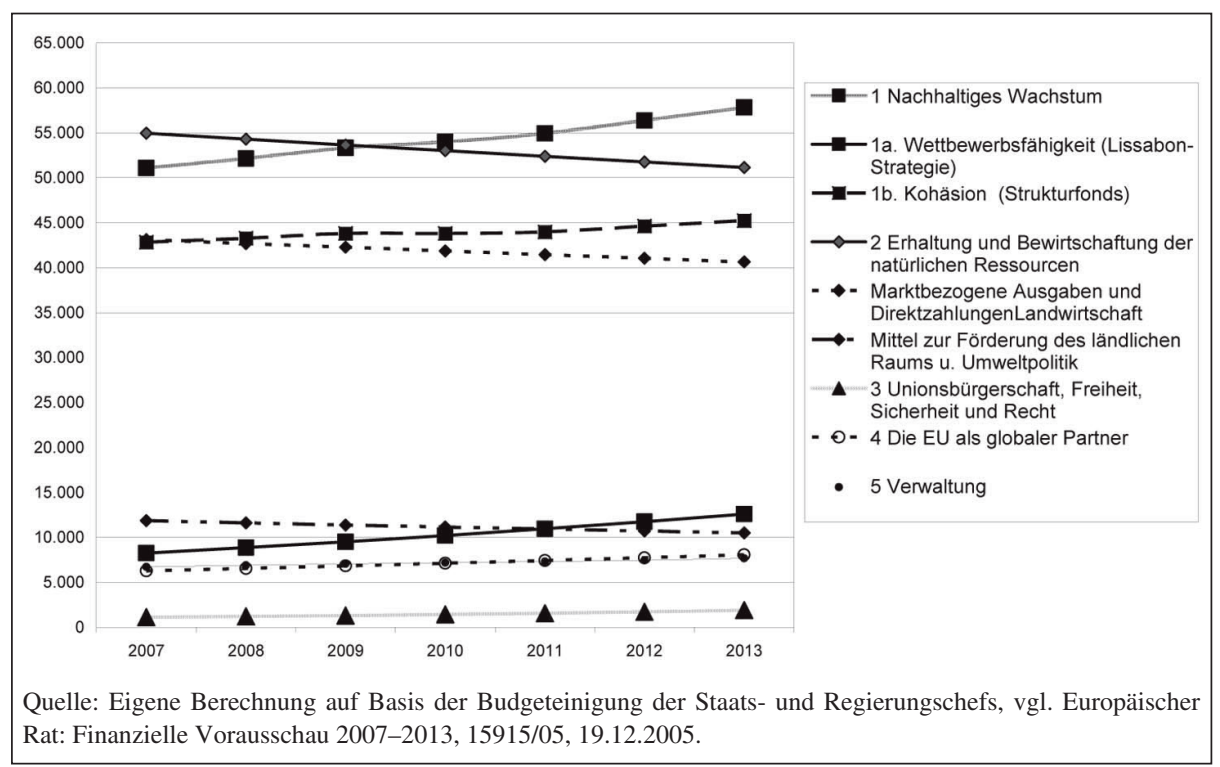

4 Vgl. „Merkels Triumph wird wohl teuer fürs Land“, in: Handelsblatt vom 23. 12. 2005 und die deutlichste Relativierung der Rolle Angela Merkels durch den Fraktionsvorsitzenden der SPE-Fraktion im Europäischen Parlament, Martin Schulz: „Das war ein Abstaubertor“, in: Der Spiegel, 3/2006, S. 46.

5 Ausführlicher hierzu Peter Becker: Mehr Geld für Europa. Die Verständigung auf einen neuen EU-Finanzrahmen, SWP-Aktuell Nr. 4, Januar 2006. 
Zwar erhalten die neuen Mitgliedstaaten gegenüber den ersten Ansätzen der Kommission weniger Gelder, jedoch müssen sie künftig nur noch einen um zehn Prozentpunkte reduzierten Kofinanzierungsanteil aus ihren nationalen Haushalten aufbringen. Als weitere Erleichterung dient die Verlängerung der Frist, innerhalb derer die in Brüssel bereitgestellten Finanzmittel abgerufen werden können, um ein Jahr auf nunmehr drei Jahre. Diese technischen Anpassungen dürften den Bedürfnissen der angespannten nationalen Haushalte und den häufig überforderten Fondsverwaltern in den neuen Mitgliedstaaten weitaus stärker entgegenkommen als eine Erhöhung der Gesamtsumme.

Eine beträchtliche Aufstockung um über 50 Prozent mit einer festgelegten jährlichen realen Steigerungsrate von 7,5 Prozent im Vergleich zu 2006 wurde für die Rubrik, Wettbewerbsfähigkeit im Dienste von Wachstum und Beschäftigung" vereinbart. Aus diesem Titel werden in erster Linie transeuropäische Netze, EU-Programme im Bereich Bildung, Forschung und Entwicklung sowie Maßnahmen der sozialpolitischen Agenda 2005-2010 im Rahmen der europäischen Beschäftigungspolitik finanziert. Den deutlichsten Zuwachs über die gesamte Laufzeit verzeichnet die Rubrik 3a ,Freiheit, Sicherheit und Recht'. Die bereitgestellten Mittel für die gemeinsame Asyl- und Einwanderungspolitik, den EU-Außengrenzschutz und die gemeinsamen Maßnahmen zur Bekämpfung der illegalen Migration, des organisierten Verbrechens und des Terrorismus sollen sich von 2007 bis 2013 mehr als verdoppeln - allerdings von einem vergleichsweise sehr niedrigen Niveau von 600 Millionen Euro im Jahr 2007 auf 1390 Millionen im Jahr 2013. Die Mittel für die Gemeinsame Außen- und Sicherheitspolitik (GASP) sollen mit einer durchschnittlichen jährlichen Wachstumsrate von 4 Prozent anwachsen. Die EU veranschlagt ungefähr genauso viel Geld für Verwaltungsausgaben wie für ihre Nachbarschaftspolitik, die Vorbeitrittshilfen und die neuen Stabilitäts- und Entwicklungsfonds (Schaubild 3).

\section{Schaubild 3: Verteilungsrelationen}

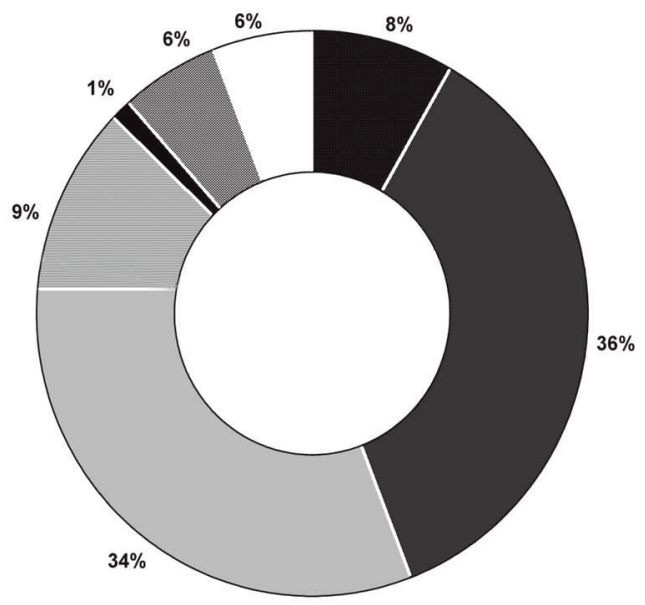

Im Uhrzeigersinn
1a. Wettbewerbsfähigkeit (Lissabon-
Strategie)
1b. Kohäsion (Strukturfonds)
$\square$ Marktbezogene Ausgaben und
DirektzahlungenLandwirtschaft
$\square$ Mittel zur Förderung des ländlichen Raums
u. Umweltpolitik
3 Unionsbürgerschaft, Freiheit, Sicherheit
und Recht
$\square 5$ Verwaltung
4 Die EU als globaler Partner

Quelle: Eigene Berechnung auf Basis der Budgeteinigung der Staats- und Regierungschefs, vgl. Europäischer Rat: Finanzielle Vorausschau 2007-2013, 15915/05, 19.12.2005. 
Im Vergleich zu den von der Europäischen Kommission vorgeschlagenen Haushaltsansätzen wurden von den Mitgliedstaaten gerade in den Rubriken Einsparungen vorgenommen, mit denen die erweiterte EU auf die neuen politischen und ökonomischen Herausforderungen der nächsten Jahre vorbereitet werden sollte. Hierzu sind zweifellos im Bereich der Justiz- und Innenpolitik die Programme zur Terror- und Kriminalprävention sowie die neuen Flüchtlings-, Grenzschutz- und Integrationsfonds zu rechnen. Trotz beträchtlicher Steigerungen zur Förderung von Forschung und Entwicklung im Rahmen der Lissabon-Strategie wurde gerade diese Hauhaltsrubrik zur eigentlichen Streichrubrik. Auch für die neuen Programme und Fonds im Bereich der GASP, wie der Solidaritätsfonds oder die neuen Stabilitäts-, Krisenreaktions- und Katastrophenschutzinstrumente sollen deutlich weniger Ressourcen bereitgestellt werden als von der Kommission veranschlagt. Hatte die Kommission im Februar 2004 davon gesprochen, dass es bei der nächsten Finanziellen Vorausschau um ein „politisches Projekt" ${ }^{\prime 6}$ gehe, das einer starken politischen Führung und einer echten politischen Vision bedürfe, so symbolisiert das Ergebnis des Europäischen Rates vom 17. Dezember 2005 die ernüchternde europäische Realität.

Zusätzlich ist der neue Finanzrahmen durch eine Inflation von Sonderregelungen zugunsten einzelner Mitgliedstaaten charakterisiert. Während der derzeitige Finanzrahmen, die Agenda 2000, 13 Sonderregelungen mit einem Gesamtvolumen von 5,265 Milliarden Euro umfasste, wird der neue Finanzrahmen insgesamt 18 Sonderregelungen mit einem Volumen von weit über 10 Milliarden Euro enthalten. Diese Bestimmungen reichen von Sonderzahlungen für einzelne Regionen wie Ceuta und Melilla, Korsika, Nordirland, Prag, die ärmsten ostpolnischen Regionen, die neuen Bundesländer, Bayern und die österreichischen Grenzregionen bis hin zu Sonderregelungen für die Aufteilung der Mittel des neuen Finanzierungsinstruments zur Förderung des ländlichen Raums (ELER) auf einige westeuropäische Mitgliedstaaten. Die größten Profiteure dieser Ausnahmeregelungen werden voraussichtlich Spanien mit Sonderzahlungen in Höhe von mindestens 2,1 Milliarden Euro, Italien mit 1,9 Milliarden Euro und Polen mit rund 1,2 Milliarden Euro sein. Mit einer Gesamtsumme von circa 55 Milliarden Euro wird Polen zum künftig größten Nettoempfänger.

Verschiedene Sonderregelungen wurden auch auf der Einnahmenseite eingeführt, um die negativen Salden der Nettozahler Österreich, Deutschland, Niederlande und Schweden zu verringern. So kalkuliert die Bundesregierung durch die Kürzung des Mehrwertsteuer-Abrufsatzes von derzeit 0,3 Prozent auf 0,15 Prozent eine Reduktion der deutschen Bruttoabführungen an den EU-Haushalt in Höhe von 1,1 Milliarden Euro jährlich. ${ }^{7}$

Die wichtigste Veränderung auf der Einnahmenseite ist aber zweifellos die Anpassung des britischen Beitragsrabatts ${ }^{8}$ an die Veränderungen in Folge der Osterweiterung. Erst nach schwierigen Verhandlungen stimmte Blair der Reform zu, um das sich verschärfende Ungleichgewicht zwischen den Nettozahlern zu mildern und den nationalen Vorteil des Sonderrabatts durch die von London stets geforderte Erweiterung der EU nicht übermäßig aus-

$6 \operatorname{KOM}(2004) 101$ „Unsere gemeinsame Zukunft aufbauen - Politische Herausforderungen und Haushaltsmittel der erweiterten Union - 2007-2013“.

7 Vgl. Antwort der Bundesregierung auf die Kleine Anfrage der Fraktion der FDP „Finanzpolitische Auswirkungen der Einigung über die Finanzielle Vorausschau 2007 bis 2013 der Europäischen Union“, in: Deutscher Bundestag, Drs. 16/566 vom 8.2.2006.

8 Die Europäische Kommission hat für die Jahre 1997 bis 2003, d.h. bezogen auf die EU-15, eine Gesamtrabattsumme von rund 30,08 Mrd. Euro zu Gunsten Großbritanniens errechnet. Aufgrund eines gesonderten Rabatts auf die Finanzierung des britischen Rabatts müssen seit 2003 Frankreich, Italien und Spanien den mit Abstand größten Anteil zu dessen Finanzierung leisten, während der Finanzierungsanteil Deutschlands von zunächst durchschnittlich rund 20 Prozent auf 8,39 Prozent im Jahr 2003 sank. Vgl. Europäische Kommission: Bericht zur Aufteilung der operativen EU-Ausgaben 2003 nach Mitgliedstaaten, September 2004, Tabelle 4d, S. 98. 
zureizen. Der Kompromiss stieß in Großbritannien auf heftige Kritik der Opposition und der Presse. ${ }^{9}$ Sah der erste britische Vorschlag zunächst lediglich eine auf den Zeitraum 20072013 begrenzte stufenweise Kürzung des britischen Rabatts um insgesamt 8 Milliarden vor, soll der Rabatt nun über die gesamte Laufzeit um maximal 10,5 Milliarden Euro gekürzt und diese Absenkung über das Jahr 2013 hinaus dauerhaft festgeschrieben werden. Damit übernimmt Großbritannien einen größeren Anteil der durch die Osterweiterung entstandenen Mehrausgaben in der EU-Strukturpolitik; lediglich die Agrarausgaben für die neuen EUStaaten werden weiterhin zugunsten Großbritanniens rabattiert.

Die deutsche Nettosaldoposition wird sich nach den Berechnungen der Bundesregierung weiter verschlechtern - von derzeit 0,36 Prozent des deutschen BNE auf circa 0,43 Prozent. Die Bruttoabführungen aus dem Bundeshaushalt an den EU-Haushalt werden von 23,8 Milliarden Euro im Jahr 2006 auf circa 28,2 Milliarden Euro im Jahr 2013 ansteigen. Zwar sinkt der deutsche Anteil an der Finanzierung des Finanzrahmens langsam von derzeit 20,6 Prozent auf 19,9 Prozent im Jahr 2013, aber dennoch wird Deutschland erneut zum größten Nettozahler in der Union werden. Als Erfolg bewertet die Bundesregierung das Zusammenrücken der großen Mitgliedstaaten im Kreis der Nettozahler; künftig werden sich Frankreich, Italien und Schweden mit jeweils 0,37 Prozent ihres BNE und Großbritannien mit 0,32 Prozent des BNE deutlich stärker an den Finanzierungslasten der EU beteiligen.

Grundsätzlich entspricht die Struktur des EU-Budgets allerdings nicht mehr den aktuellen Anforderungen an ein gemeinschaftliches Handeln und eine entsprechende gemeinsame Finanzierung. Auch das eingeübte Verhandlungsverfahren verhindert durch die Konzentration auf die unbedingt erforderliche Lösung einer komplexen Verhandlungssituation eine grundlegende Prüfung und Anpassung der europäischen Finanzverfassung. Die kritische Verhandlungskonstellation im Jahr 2005 hat gezeigt, dass in der erweiterten Europäischen Union mit ihren eklatanten ökonomischen und sozialen Disparitäten dieses System künftig nicht mehr so leicht zu einvernehmlichen Lösungen führt, sondern vielmehr die bestehenden Konflikte weiter vertieft. ${ }^{10}$

\section{Der Haushaltsacquis - Themen und Verlauf der Verhandlungen}

In den Verhandlungen über die Finanzielle Vorausschau werden sowohl die Ausgabenplafonds der einzelnen Haushaltsrubriken festgelegt als auch das maximal zur Verfügung stehende Volumen der Jahreshaushalte fixiert. Die jährlich nach dem Verfahren des Artikels 271 EGV im Zusammenwirken von Rat und Parlament aufzustellenden EU-Jahreshaushalte müssen sich in dieses Tableau der Finanziellen Vorausschau einfügen. Im Vergleich zu den schwierigen und für Konflikte zwischen Rat und Parlament anfälligen System der Jahreshaushalte hat sich bislang die mehrjährige Finanzplanung bewährt. Die gemeinsame und für alle Verhandlungspartner verbindliche Festlegung von Ausgabenobergrenzen verhindert die für die siebziger und achtziger Jahre charakteristischen Budgetkonflikte. Somit garantiert der Finanzrahmen eine größere finanzpolitische Stabilität und ein hohes Maß an haushaltspolitischer Vorhersehbarkeit.

9 Vgl. „EU rebate deal will cost $£ 1.9$ bn a year by 2010“, in: Financial Times vom 20.12.2005; Kai Oppermann: Die britische Ratspräsidentschaft 2005: Zwischen europäischen Erwartungen und innenpolitischen Restriktionen, in: integration, 1/2006, S. 23-37.

10 Nicht ohne Grund sagte der österreichische Bundeskanzler Wolfgang Schüssel unmittelbar nach dem Gipfel: „Das nächste Mal werden wir uns an die Gurgel gehen.“Vgl. Süddeutsche Zeitung vom 31. 12. 2005. 


\section{Das Verhandlungspaket im Überblick}

Das Verhandlungspaket der Agenda 2007 setzt sich, ebenso wie bei den früheren Finanzverhandlungen, im Wesentlichen aus drei Komponenten zusammen:

- Die Finanzielle Vorausschau und das zu ihrer Implementierung entwickelte Instrument der Interinstitutionellen Vereinbarung (IIV) als Teil des europäischen soft law.

Die Finanzielle Vorausschau entfaltet ihre rechtliche Bindungswirkung nicht in den einvernehmlichen Schlussfolgerungen des Europäischen Rats, mit denen das Finanzpaket politisch verbindlich verabschiedet wird, sondern erst mit der IIV ${ }^{11}$ zwischen Rat, Kommission und Europäischem Parlament. Auch die neue Finanzielle Vorausschau bedarf dieser besonderen europarechtlichen Form, damit auch das Europäische Parlament als gleichberechtigter Teil der EU-Haushaltsbehörde auf die Vorgaben beziehungsweise die Grenzen des Finanzrahmens verpflichtet wird.

- Ein neuer Eigenmittelbeschluss

Die Veränderung des Einnahmensystems der EU, wie zum Beispiel die vereinbarte Modifizierung des britischen Beitragsrabatts, erfordert einen neuen Eigenmittelbeschluss nach Art. 269 EGV, der einstimmig verabschiedet und von den nationalen Parlamenten ratifiziert werden muss.

- Die Legislativvorschläge der Europäischen Kommission zur Umsetzung der haushaltspolitisch festgelegten Prioritäten in konkrete Programme und Fonds.

Erst die Verordnungstexte bilden die Rechtsgrundlage der EU-Ausgabenprogramme in den verschiedenen Sachpolitiken. Nahezu 90 Prozent der mehrjährigen Ausgabenprogramme laufen Ende 2006 aus und bedürfen einer neuen Rechtsgrundlage, damit sie auch 2007 fortgeführt werden können. Die Mehrzahl müssen im Mitentscheidungsverfahren (mit dem Europäischen Parlament) verabschiedet werden.

Grundsätzlich gilt, dass das Finanzpaket im Konsens aller Mitgliedstaaten entschieden wird. Dadurch verfügt jeder Mitgliedstaat über eine starke Veto-Position, die weit über das relative Verhandlungsgewicht bei den üblichen Gesetzgebungsverfahren hinaus reicht. Zwar ist eine Verständigung auf die Verordnungen des Legislativpakets auch ohne eine Einigung auf das Finanzpaket denkbar, aber erst in diesen Finanzverhandlungen werden die Haushaltsansätze für die jeweiligen Politikbereiche definiert. Weil die beiden Komponenten des Gesamtpaktes überaus eng miteinander zusammenhängen, ist allen Beteiligten bewusst, dass auch das Legislativpaket zwar differenziert im Rat und gemäß dem jeweiligen Entscheidungsverfahren im Zusammenspiel mit dem Europäischen Parlament verhandelt wird, aber eine Einigung nur im Gesamtzusammenhang möglich ist. Diese Abhängigkeit der Legislativverfahren von der Verständigung auf das Gesamtvolumen des Finanzrahmens wurde von den Mitgliedstaaten nach einem Treffen der Präsidenten von Kommission, Parlament und Rat Anfang September 2005 nochmals explizit bekräftigt. ${ }^{12}$

11 Die IIV selbst ist im europäischen Primärrecht lediglich durch die Erklärung (Nr. 6) zu Artikel 100 des EGV im Vertrag von Nizza verankert. Der rechtliche Status, bzw. die Rechtsverbindlichkeit der IIV ist noch ungeklärt, weil bislang kein Urteil des EuGH diese Frage beantwortet hätte. Allerdings hat der EuGH in mehreren Urteilen - zuletzt in seiner Rechtsprechung zur Abgrenzung von obligatorischen und nicht-obligatorischen Ausgaben und dem damit zusammenhängenden zum Höchstsatzverfahren des Art. 203 Absatz 9 EGV vom Dezember 1995 - auf die besondere Bedeutung der Einigung zwischen Rat und EP hingewiesen. Auch der EuGH misst dem Konsens aller Organe, wie er sich in der IIV am deutlichsten manifestiert, demnach einen hohen rechtlichen Wert zu.

12 Vgl. Note from the Presidency to the Coreper: Handling the legislative proposals for the Financial Perspective 2007-13, in : Dokument 1270/1/05, Brüssel, vom 15. September 2005. 


\section{Verhandlungsthemen und Konfliktlinien}

Die komplexe Verhandlungssituation erfordert stets eine schwierige Koordinierung der verschiedenen Verhandlungsarenen und -gruppen. Sie ermöglicht allerdings nicht nur eine Vielzahl von Blockademöglichkeiten, sondern andererseits auch die Möglichkeit zu Koppelgeschäften, side payments und Paketlösungen zwischen einzelnen Politiken und Verhandlungsarenen. Die antagonistischen Interessen der Mitgliedstaaten lassen sich entlang der drei nahezu klassischen Konfliktlinien einordnen, die auch diese Verhandlungsrunde charakterisierten:

- Die Auseinandersetzung über die Gesamtsumme der Finanziellen Vorausschau, die dominiert wurde vom Streit über die nationalen Bilanzen von Einzahlungen in und Rückflüssen aus dem EU-Haushalt, das heißt der Konflikt zwischen Nettozahlern und Nettoempfängern unter den Mitgliedstaaten.

- Dem Konflikt über die Verteilung der Finanzmittel auf die einzelnen Haushaltsrubriken, der bestimmt wurde von der Diskussion über die Ausstattung der Gemeinsamen Agrarpolitik und die Frage der Solidarität zwischen den Mitgliedstaaten.

- Dem Konflikt über eine gerechte und transparente Finanzierung der Union, bei denen die Fortführung des britischen Beitragsrabatts im Mittelpunkt stand.

Bei den Finanzverhandlungen standen sich demnach die Festschreibung von Haushaltsdisziplin und Beitragsgerechtigkeit auf der einen Seite und das Einfordern von finanzieller Solidarität sowie ökonomischer und sozialer Kohäsion in der EU auf der anderen Seite gegenüber. Jedoch wurde die Verhandlungskonstellation durch eine besondere Situation zusätzlich erschwert: Die größten Beitragszahler der EU haben als Folge der anhaltenden ökonomischen Wachstumsschwäche und der angespannten nationalen Haushalte Finanzierungsprobleme, während andererseits gerade durch die Erweiterung um die vergleichsweise ärmeren Mitgliedstaaten aus Mittel- und Osteuropa der Verteilungsbedarf in der EU deutlich angewachsen ist und die Forderung nach einer Budgetaufstockung umso lauter vorgebracht wurde.

Ebenso bedeutsam wie die zentralen Themen und die charakteristischen Konfliktlinien im Verhandlungsprozess waren auch die Fragen, die in den Verhandlungen nicht oder nur unzureichend aufgegriffen wurde. Betrachtet man die Fragen, die nicht Verhandlungsgegenstand waren, dann wird die Kluft zwischen dem Reformbedarf und den Verhandlungssackgassen, in die sich die Realpolitik manövriert hat, deutlich. Nicht verhandelt wurde sowohl über Veränderungen des Verhandlungs- und Entscheidungsverfahren als auch über eine grundlegende Reform des europäischen Eigenmittelsystems oder die Neuausrichtung der Ausgabenpolitik. So spielte der Vorschlag des EU-Konvents, die Laufzeiten der Finanziellen Vorausschau an das Mandat der Kommission und die Legislaturperiode des Europäischen Parlaments anzugleichen, keine Rolle. Die Parallelität der Lauf- und Mandatsperioden sollte sowohl die Europäische Kommission als Initiator von ausgabenwirksamen Programmen als auch das Parlament als Teil der Haushaltsbehörde stärker in die politische Verantwortung für die Schwerpunktsetzung der Finanziellen Vorausschau nehmen und damit das Verfahren insgesamt parlamentarischer und demokratischer gestalten. Ebenso wenig wurde, trotz einiger Anstöße der Kommission und der Mitgliedstaaten ${ }^{13}$, eine umfassende Um-

13 Die Europäische Kommission hatte zwar den Bewertungsmaßstab des „europäischen Mehrwert“ in ihrer Mitteilung zur Finanziellen Vorausschau vom 14. Juli 2004 angelegt, um die von ihr vorgeschlagen Ausgabensteigerungen zu rechtfertigen, und auch der Europäische Rat hatte in seinen Schlussfolgerungen vom 16./17. Dezember 2004 gefordert, dass alle finanzwirksamen Maßnahmen der EU nicht nur im Einklang mit den allgemeinen Grundsätzen der Subsidiarität, der Verhältnismäßigkeit und der Solidarität stehen müssen, sondern darüber hinaus diese Maßnahmen auch einen zusätzlichen Nutzen erbringen müssen. 
schichtung der Ausgabenschwerpunkte des künftigen Finanzrahmens auf Maßnahmen und Programme verhandelt, die einen europäischen Mehrwert versprechen. Ein solcher zusätzlicher europäischer Nutzen wird grundsätzlich von europäischen öffentlichen Gütern zu erwarten sein, deren Vorteile allen Mitgliedstaaten zugute kommen sollten. Welche das sein könnten, darüber bestehen unterschiedliche Vorstellungen und Interessen ${ }^{14}$ - angefangen im Bereich Forschung und Entwicklung, Bildung und Ausbildung sowie der Verbesserung der europaweiten Infrastruktur bis hin zu einer europäischen Sicherheits- und Verteidigungspolitik, der Schaffung eines europäischen Verteidigungsfonds, eine gemeinsame europäische Entwicklungspolitik oder die gemeinsame Gewährleistung der inneren Sicherheit und die Finanzierung eines EU-Außengrenzschutzes. ${ }^{15}$

\section{Die drei Phasen des Verhandlungsprozesses}

Am Beginn des Verhandlungsprozesses zu einer Finanziellen Vorausschau steht üblicherweise die Mitteilung der Kommission, in der sie ihre strategischen und finanziellen Ziele sowie ihre politischen Schwerpunkte vorstellt. ${ }^{16}$ Diesem offiziellen Verhandlungsauftakt zur Finanziellen Vorausschau 2007-2013 ging zwischen April und Juli 2003 ein intensives internes Abstimmungsverfahren innerhalb der Kommission voraus, in das alle Generaldirektionen eingebunden waren. ${ }^{17}$ Die internen Diskussionen hatten zwischen den Kommissionsdienststellen zu teils kontroversen Verhandlungen über die Haushaltsansätze und Ausgabenprioritäten geführt und waren ein Grund für die mehrmalige Verschiebung der Verabschiedung der Mitteilung. ${ }^{18}$

Die Kommission wählte einen ,bottom up-approach` und versuchte ihre Vorschläge für die spezifischen Haushaltsansätze den politischen Zielen der erweiterten EU anzupassen. Dieser Ansatz definierte zunächst die anstehenden Herausforderungen und die von den Mitgliedstaaten auf die EU übertragenen Aufgaben. Erst in einem zweiten Schritt errechneten die Dienststellen der Kommission die benötigten Haushaltsmittel, um alle übertragenen Aufgaben erfüllen zu können. Dagegen plädierte die Gruppe der Nettozahler im Kreis der Mitgliedstaaten für ein entgegengesetztes Verfahren (,top down-approach ${ }^{6}$ ), der in einem ersten Schritt die Deckelung der Ausgaben auf 1 Prozent des EU-BNE vorsah und erst anschließend

14 Vgl. u.a. André Sapir: An agenda for a growing Europe. Making the EU Economic System deliver, Juli 2003; Daniel Gros und Stefano Micossi: A better budget for the European Union. More Value for money, more money for value, in: CEPS policy brief Nr. 66, Februar 2005; Friedrich Heinemann und Maxime Lefebvre: Deutschfranzösische Überlegungen zur Zukunft der EU-Haushalts, in: DGAP-Analyse Nr. 31, November 2004.

15 Vgl. für eine politik-ökonomische Argumentation u.a. Lars P. Feld: Viel Lärm um nichts? Die Agenda 2007 der EU aus ökonomischer Sicht, in: integration 1/2005, S. 55-67; Cay Folkers: Welches Finanzausgleichsystem braucht Europa?, in: Helmut Karl/Wilhelm Henrichsmeyer (Hrsg.): Regionalentwicklung im Prozeß der Europäischen Integration, Bonn 1995, S. 87-108.

16 Vgl. zu dieser Phase der Agenda 2007 - Verhandlungen Peter Becker: Die Agenda 2007. Die erste Etappe der europäischen Finanzverhandlungen 2007-2013, in: SWP-Aktuell 34, August 2004.

17 Die Kommission hatte entsprechend den fünf für die Agenda 2007 vorgeschlagenen Haushaltsrubriken fünf übergreifende Arbeitsgruppen gebildet, die gestützt auf eine Analyse der anstehenden europapolitischen Herausforderungen und der bereits formulierten politischen Prioritäten der erweiterten Union eine Zusammenstellung der benötigten Haushaltsmittel erarbeiteten. Hinzu kam eine sechste Arbeitsgruppe zur Einnahmeseite des EU-Haushalts. Die Berichte der Arbeitsgruppen sind im Internet abrufbar unter: http://europa.eu.int/comm/ financial_perspective/documents_commission/index_en.htm, (letzter Zugriff: 08.03.2006).

18 Die Diskussion innerhalb der Kommission war durch die Studie der Forschergruppe um André Sapir im Auftrag des Kommissionspräsidenten Romano Prodi verschärft worden, von der sich der damalige Kommissar für Regionalpolitik Michel Barnier deutlich distanzierte. Rainder Steenblock und Ines Hartwig bewerten die Konflikte in der Kommission dementsprechend drastisch: „Als ein Novum in der Geschichte der europäischen Integration haben die Kommissare sich bei einem für die Europäische Union derart wichtigen Reformpaket offen auseinander dividieren lassen." Rainder Steenblock/Ines Hartwig: Die Agenda 2007: Solidarität, Nachhaltigkeit und Innovation, in: integration 1-2/2004, S. 85-94, S. 85. 
die Prioritäten in diesem so begrenzten Finanzrahmen definierte. Mit einem gemeinsamen Schreiben an EU-Kommissionspräsident Romano Prodi hatten sechs Staats- und Regierungschefs bereits am 15. Dezember 2003 den Verhandlungsprozess eröffnet. In ihrem Schreiben erklärte diese Gruppe der sechs Nettozahler Deutschland, Frankreich, Großbritannien, Niederlande, Österreich und Schweden, dass sie ,keinen Spielraum für einen EU-Haushalt in der Nähe der derzeitigen Eigenmittelobergrenze“ von 1,24 Prozent des EU-BNE sehen.

Der offizielle Verhandlungsauftakt erfolgte am 10. Februar 2004 mit der Mitteilung „Unsere gemeinsame Zukunft aufbauen: Politische Herausforderungen und Haushaltsmittel der erweiterten Union 2007-2013“, in der die Kommission einen Finanzbedarf von insgesamt 1.025,026 Milliarden Euro errechnete. Am 14. Juli 2004 konkretisierte sie diese erste Mitteilung durch ein erstes Paket von Gesetzgebungsvorschlägen und durch drei weitere Mitteilungen. Dieses Paket umfasste Rechtsverordnungen zur künftigen Kohäsions- und Strukturpolitik, zwei Vorschläge zur Reform des europäischen Eigenmittelsystems und zur Überprüfung des britischen Sonderrabatts sowie den Entwurf einer neuen IIV. ${ }^{19}$ Die Kommission vervollständigte mit zwei weiteren Legislativpaketen am 29. September 2004 und am 6. April 2005 das Gesamtpaket der Agenda 2007. Im weiteren Verfahrensablauf schwand jedoch ihre Verhandlungsstärke, und ihr Einfluss wurde nur noch mittelbar über die Aggregation von Daten und Zahlen zur Begleitung der Verhandlungen zwischen den Mitgliedstaaten spürbar. So legte die Kommission auf Anfrage der Mitgliedstaaten für die Verhandlungen im Rat mehr als 90 Hintergrundpapiere, sogenannte fiches thematiques, vor, die allen Mitgliedstaaten als unabhängige Bezugs- und Berufungsgrundlage dienten.

In dieser zweiten Phase dominierten die Mitgliedstaaten im Rat und dessen Arbeitsgruppen den Verhandlungsprozess. Die unterschiedlichen Elemente des Legislativpakets wurden zeitgleich in den betroffenen Fachräten verhandelt, wobei dem Rat, Allgemeine Angelegenheiten " eine koordinierende Rolle zukam. Im Vordergrund standen zweifellos die Verhandlungen über die Finanzielle Vorausschau, die den Rahmen für alle anderen Verhandlungsagenden lieferten.

Nach der Vorlage der Mitteilung der Kommission nahmen die Mitgliedstaaten zunächst eine allgemeine Bewertung des Kommissionsvorschlages vor. Hierfür wurde auf Arbeitsebene eine ad hoc-Arbeitsgruppe Finanzielle Vorausschau gebildet, die unter luxemburgischer Präsidentschaft von der Gruppe ,Freunde der Präsidentschaft‘ abgelöst wurde. Beide Gruppen konzentrierten ihre Verhandlungen auf die Finanzaspekte der Agenda 2007, während die Facharbeitsgruppen des Rates die Legislativvorschläge für die Politikbereiche verhandelten. Nach der Phase der allgemeinen Aussprache wurde unter niederländischer Präsidentschaft eine detaillierte inhaltliche Prüfung der einzelnen Haushaltsrubriken des Finanzrahmens vorgenommen. Dabei verfolgte die Präsidentschaft einen , building bloc-approach', dessen Ziel eine differenzierte Analyse der spezifischen Ausgabenblöcke und damit verbunden eine vertiefte Debatte über die veranschlagten Ausgabensummen war. Drei Maßstäbe dienten als Bewertungskategorien ${ }^{20}$ :

$19 \operatorname{KOM}(2004) 487$ „Finanzielle Vorausschau 2007 - 2013“; KOM(2004) 498 „,Vorschlag zur Erneuerung der Interinstitutionellen Vereinbarung über die Haushaltsdisziplin und die Verbesserung des Haushaltsverfahrens"; $\operatorname{KOM}(2004) 505$,Technischer Anhang - Finanzierung der Europäischen Union: Bericht der Kommission über das Funktionieren des Eigenmittelsystems".

20 Die niederländische Präsidentschaft definierte die Funktion des ,building bloc-approach“ so: „Dieser Ansatz besteht darin, die Beratungen auf eine breitere Grundlage zu stellen, indem der Inhalt der verschiedenen Politikbereiche und die daraus resultierende Mittelausstattung in den Mittelpunkt gerückt wird, um auf diese Weise verschiedene politische Optionen und die jeweils damit verbundene finanziellen Auswirkungen ermitteln zu können." Vgl. Sachstandsbericht des Vorsitzes, in: Dokument 14953/04 vom 19. November 2004, Ziffer 2, S. 2. 
- die Effektivität einer vorgeschlagenen Maßnahme: die Prüfung, ob ein Tätigwerden der EU zu einem europäischen Mehrwert und zu besseren Ergebnissen führt;

- die Effizienz: ob eine Maßnahme auf EU-Ebene Kosteneinsparungen auch für die nationalen Haushalte ermöglicht;

- die Synergieeffekte: ob die gegenseitige Ergänzung von Maßnahmen der EU und der Mitgliedstaaten sinnvoll ist.

Diese Verlagerung des Blickwinkels von den fiskalischen auf die materiellen Fragen des Finanzrahmens machte den Austausch über die Ziele einzelner Politiken, den zusätzlichen Nutzen dieser Politiken für die Union und die Mitgliedstaaten sowie die notwendigen Finanzmittel erforderlich. Ein besonderer taktischer Vorteil dieser Form der Diskussion im Ministerrat lag auch darin, dass die Vorschläge der Kommission, deren Prioritätensetzung und die vorgeschlagenen Einzelmaßnahmen analysiert, hinterfragt und damit zugleich relativiert wurden. Am Ende der niederländischen Präsidentschaft waren die Vorschläge und der Entwurf eines Finanztableaus der Kommission nicht mehr die alleinige Verhandlungsgrundlage. Die Vorschläge und Finanzansätze der Mitgliedstaaten, insbesondere der Gruppe der sechs Nettozahler, traten gleichrangig daneben. ${ }^{21}$

Die nachfolgende luxemburgische Präsidentschaft versuchte, in einem weiteren Schritt die fragmentierte Diskussion über einzelne Politikfelder und verschiedene Haushaltsrubriken wieder zusammen zu führen und einem Gesamtkompromiss näher zu bringen. Das Kerninstrument dieser Politik war die sogenannte, negotiation box ${ }^{622}$. In diesem kontinuierlich überarbeiteten non-paper versuchte die Präsidentschaft im Format möglicher Schlussfolgerungen des Europäischen Rats die Verhandlungen deutlicher auf einen Gesamtkompromiss zu fokussieren und ihnen eine gewisse Dynamik zu verleihen. Sie nutzte das Instrument dazu, um Kompromissbereitschaft einzufordern, die ohne diesen Anstoß nicht zu erzielen gewesen wäre. Erst kurz vor dem Treffen der Staats- und Regierungschefs am 16./17. Juni 2005 hatte die luxemburgische Präsidentschaft am 2. Juni 2005 ein Verhandlungspapier vorgelegt, in dem erstmals konkrete Zahlen für eine Finanzielle Vorausschau enthalten waren. Der Vorschlag sah für den siebenjährigen Geltungszeitraum der Finanziellen Vorausschau Ausgaben in Höhe von insgesamt 875 Milliarden Euro vor. Dies sollte 1,06 Prozent des EU-BNE entsprechen und damit näher bei dem von der Gruppe der sechs Nettozahler geforderten Gesamtrahmen von 1,0 Prozent des EU-BNE oder 815 Milliarden Euro liegen als am ursprünglichen Vorschlag der Kommission. Der Vorschlag sah Einsparungen insbesondere in der Haushaltsrubrik, Wettbewerbsfähigkeit im Dienste von Wachstum und Beschäftigung' gegenüber dem Vorschlag der Kommission vor. Zugleich blieben die Ausgaben für die Direktzahlungen an die Landwirte und für die Marktordnungen unangetastet. Von besonderer Brisanz war der Vorschlag, den Beitragsrabatt Großbritanniens auf einem Niveau von 4,6 Milliarden Euro einzufrieren und dann ab dem Jahr 2007 schrittweise langsam abzuschmelzen. Zugunsten der übrigen Nettozahler schlug die Präsidentschaft einen gesonderten Rabatt im Bereich der Mehrwertsteuereigenmittel vor.

Um einen Ausgleich der gegenläufigen Interessen der größten Nettozahler - Deutschland, die Niederlande, Schweden und Österreich auf der einen Seite - und den hauptsächlich Begünstigten der bisherigen EU-Ausgabenpolitik - Frankreich und Spanien - auf der ande-

21 In dem Abschlußbericht der niederländischen Präsidentschaft zu den ,Bausteinen “ wird diese Gleichrangigkeit verschiedener Ansätze besonders deutlich. Vgl. Vermerk des Vorsitzes, Dokument 14952/04 vom 19. November 2004 .

22 Damit griff die luxemburgische Präsidentschaft ein Verfahren der deutschen Präsidentschaft zur Agenda 2000 im Frühjahr 1999 auf. In ihren einleitenden Bemerkungen stellte die Präsidentschaft diesen Rückgriff auf ein einmal eingespieltes Verfahren explizit heraus. 
ren Seite zu ermöglichen, hatte die Präsidentschaft versucht, den erforderlichen finanziellen Spielraum durch den Abbau des britischen Sonderrabatts zu schaffen. Die britische Regierung sah aber keine Möglichkeit, dieser einschneidenden Verschlechterung des britischen Status quo zuzustimmen, ohne dabei gleichzeitig den Status quo der anderen Begünstigten, insbesondere Frankreichs, in gleichem Umfang, mit gleicher Endgültigkeit und vergleichbarem öffentlichem Aufmerksamkeitswert anzutasten. Nach dem Scheitern des Juni-Gipfels wurden die Verhandlungen erst mit einem Konklave der Außenminister am 7. Dezember 2005 wieder aufgenommen. Zuvor hatte der britische Vorsitz in bilateralen Vorgesprächen versucht, mögliche Verhandlungsspielräume auszuloten. Nach einer nochmaligen Verringerung des Gesamtvolumens, verbunden mit den beschriebenen technischen Anpassungen, sowie einer weiteren Zuspitzung der Verhandlungskonstellation durch die britische ,Hinhaltetaktik' gelang schließlich der Kompromiss am 17. Dezember 2005.

Die Verständigung des Europäischen Rats bildet den Kulminationspunkt und Abschluss der Verhandlungen im Kreis der Mitgliedstaaten, aber noch nicht das Ende des Gesamtprozesses. Nun muss diese politische Verständigung durch eine IIV zwischen der Europäischen Kommission, dem Rat und dem Europäischen Parlament rechtlich verbindlich fixiert werden. Damit eröffnet sich eine neue Verhandlungsarena, die durch die direkte und gleichberechtigte Einbeziehung des Europäischen Parlaments in den Verhandlungsprozess gekennzeichnet ist. Der Spielraum des Parlaments für Modifikationen bleibt dennoch begrenzt - gerade auch angesichts der hohen politischen Kosten, den einmal gefundenen Gesamtkompromiss im Kreis der Mitgliedstaaten aufzulösen und neu zu verhandeln. Die deutliche Ablehnung des Verhandlungsergebnisses am 12. Januar 2006 im Europäischen Parlament zeigt jedoch, dass die Parlamentarier auch bei dieser Verhandlungsrunde nicht bereit sind, alle Kompromisse des Europäischen Rats unwidersprochen mitzutragen. ${ }^{23}$

Obwohl das Parlament zunächst nicht an den Verhandlungen beteiligt war, hatten die Parlamentarier frühzeitig ihre besonderen Forderungen für die Agenda 2007 formuliert. Nach einer ersten vorläufigen Positionierung vom 20. April 2004 verabschiedete das neugewählte Europäische Parlament am 8. Juni 2005 eine ausführliche Entschließung ${ }^{24}$, in der es seine Vorstellungen und Forderungen für die Finanzverhandlungen zusammenfasste. Darin forderten die Europaabgeordneten mehr Geld für die Verwirklichung der Lissabon-Ziele, die gemeinsame Innen- und Justizpolitik und die GASP. Weniger Ressourcen sollten für die Direktzahlungen im Bereich der Landwirtschaft aufgewendet werden; hier schlug das Parlament sogar den Einstieg in die Kofinanzierung vor. Insgesamt sollten nach den Wünschen des Parlaments rund 975 Milliarden Euro für die Agenda 2007 veranschlagt werden. Darüber hinaus stellte das Parlament eine direkte Verbindung zwischen der Zustimmung zum Gesamtpaket der Finanziellen Vorausschau und einer ,tiefgreifenden Reform des gegenwärtigen Systems der Eigenmittel der EU“ "her und forderte deshalb, dass in die neue IIV eine ,Revisionsklausel " aufgenommen werden sollte. Zuletzt formulierten die Europaparlamentarier am 1. Dezember 2005 vier ,nicht verhandelbare" Positionen ${ }^{25}$, wie einen Revisionsmechanismus, ein neues mehrjähriges Flexibilitätsinstrument, das rund 0,03 Prozent des EU-BNE beziehungsweise insgesamt rund 20 Milliarden Euro betragen soll, die Revision der Haushaltsordnung und Einführung des Mitentscheidungsverfahrens bei den neuen EU-Programmen im Bereich der GASP.

23 Vgl. ausführlicher zur Rolle des Europäische Parlaments bei den Finanzverhandlungen Peter Becker: Das Europäische Parlament in den EU-Finanzverhandlungen, in: Andreas Maurer/Dietmar Nickel (Hrsg.), Das Europäische Parlament. Supranationalität, Repräsentation und Legitimation, Baden-Baden 2005, S. 179-199.

24 Entschließung des Europäischen Parlaments zu den politischen Herausforderungen und Haushaltsmitteln der erweiterten Union 2007-2013, P6_TA-PROV(2005)0224, Berichterstatter: Reimer Böge.

25 Entschließung des Europäischen Parlaments zu der Interinstitutionellen Vereinbarung über die Haushaltsdisziplin und die Verbesserung des Haushaltsverfahrens, P6_TA-PROV(2005)0453. 


\section{Ausblick}

\section{Die Revisionsklausel}

Um sowohl den Forderungen der Europaparlamentarier als auch einiger Mitgliedstaaten nach einer umfassenden Reform des EU-Finanzsystems entgegenzukommen (neben Großbritannien hatte auch Schweden auf einer solchen Grundsatzreform bestanden), enthält der Kompromiss des Europäischen Rats vom 17. Dezember 2005 eine spezielle Überprüfungsklausel. Danach wollen die Staats- und Regierungschefs im Jahr 2009 eine umfassende „Neubewertung des Finanzrahmens“ durchführen, die sowohl die Einnahmen- als auch die Ausgabenseite des EU-Haushalts einschließt. Die Kommission wird aufgefordert, bis 2008/ 09 ,eine vollständige, weit reichende Überprüfung vorzunehmen“. Wichtig ist, dass in der Überprüfungsklausel sowohl die Gemeinsame Agrarpolitik als auch der britische Beitragsrabatt explizit genannt werden. ${ }^{26}$ Die Verbindung dieser beiden Reformschwerpunkte auf der Einnahmen- und der Ausgabenseite kann, wie Tony Blair in seiner letzten Rede als Ratspräsident am 20. Dezember 2005 vor dem Europäischen Parlament betonte, zu einer umfassenden und dauerhaften Reform der Strukturen des EU-Finanzsystems führen. Die Kommission werde jedenfalls, so Kommissionspräsident Barroso, in ihre Überprüfung alle Haushaltskategorien und Politikbereiche einbeziehen und im Jahr 2008 ein Weißbuch vorlegen. Ohne den Begriff der EU-Steuer ausdrücklich zu verwenden, forderte Barroso eine autonome Eigenmittelquelle der EU, um künftig von den schwierigen Haushaltsverhandlungen unabhängiger zu werden.

Dennoch bleibt die Formulierung der Revisionsklausel überaus unbestimmt. Eckpunkte und Zielvorgaben für die vorgesehene umfassende Reform des EU-Finanzrahmens werden darin nicht benannt. Die Unbestimmtheit der Klausel und die Tatsache, dass der neue Finanzrahmen bis zum Jahr 2013 festgeschrieben ist, eröffnet zwei denkbare, aber gegensätzliche Szenarien:

- Szenario 1: Die Kommission nutzt die offene Formulierung der Klausel, um frühzeitig einen breit angelegten öffentlichen Reformdiskurs zu beginnen, bei dem der europäische Mehrwert gemeinsamer Politiken und deren ausreichende Finanzierung im Mittelpunkt stehen. Partner in dieser Debatte wären das Europäische Parlament, einige Mitgliedstaaten und deren nationale Parlamente. Allerdings muss eine solche ,Reformkoalition ' die nach der Erfahrung des Beinahe-Scheiterns dieser Verhandlungsrunde aktuell spürbare Reformbereitschaft bis zum Jahr 2009 am Leben erhalten, um einem Rückfall in die bekannten Verhaltensmuster vorzubeugen.

- Szenario 2: Die Revision 2009 wird von den Mitgliedstaaten als unverbindliche Vorphase zu den eigentlichen, im Jahr 2011 beginnenden Etatverhandlungen für den übernächsten Finanzrahmen 2014-2020 betrachtet. Ohne zeitlichen und sachlichen Einigungsdruck verhandeln die Mitgliedstaaten gänzlich ohne Reformambitionen entlang nationaler Maximalpositionen und diskutieren in bedeutungslosen Grundsatzverhandlungen das Weißbuch der Europäischen Kommission. Jede mögliche Veränderung des Status quo messen sie an den Auswirkungen auf das nationale Nettosaldo.

26 Vgl. Iain Begg/Friedrich Heinemann: New budget, old dilemmas, in: Centre for European Reform, Briefing note, Februar 2006. 


\section{Die Nettosaldo-Logik als Kernproblem der fehlenden Reformfähigkeit}

Noch immer messen die Mitgliedstaaten den Wert der europäischen Integration in den Verhandlungen über die Finanzielle Vorausschau hauptsächlich nach ihrem Nettosaldo. ${ }^{27}$ Die Europäische Kommission akzeptierte das Thema der Nettosalden erstmals im Oktober 1998 und gab damit dem heftigen Drängen der Nettozahler nach. Seither ist das traditionelle mitgliedstaatliche Muster, die eigene Verhandlungsposition während der Finanzverhandlungen entlang der errechneten Nettosaldoposition auszurichten, auch formal von der Europäischen Kommission anerkannt worden. ${ }^{28}$

Diese Fokussierung auf die nationalen Nettosaldobilanzen ist jedoch zur Hauptursache der Unzulänglichkeiten, Ineffizienz, Komplexität und Intransparenz der bestehenden europäischen Finanzordnung geworden. Sie führt zu falschen politischen Prioritätensetzungen und langfristig zu nicht angemessenen Politikergebnissen für alle Akteure. Eine Konsequenz dieser Politik ist es, dass im bestehenden Eigenmittelsystem ebenso wie in der europäischen Ausgabenpolitik eine Vielzahl von Anpassungsmöglichkeiten inhärent verankert wurde, um auf nationale Sonderwünsche zur Anpassung der Nettopositionen reagieren zu können. Mit jedem der drei bislang verabschiedeten Finanzpakete nahm die Unübersichtlichkeit des europäischen Finanzierungssystems zu. Jede der drei Finanzierungsquellen ist mit einer ganzen Reihe von Ausnahmen und Sonderregelungen verbunden, die zwar die schwierige Kompromissfindung bei den Verhandlungen über eine Finanzielle Vorausschau erleichterten. Sie entsprechen aber keineswegs dem Gebot der Transparenz, der Überschaubarkeit und der Verständlichkeit. Die vorrangige Funktion der Sonderregelungen ist es vielmehr, als ,Stellschrauben' einen Ausgleich zwischen den nationalen Interessen der Mitgliedstaaten zu ermöglichen. Die Folge dieser systemisch angelegten Tendenz zur Intransparenz ist die vorrangige Orientierung aller Akteure auf die Auflösung komplizierter Verhandlungssituationen. Nachrangig sind hingegen die Effizienzsteigerung, die gerechte Aufteilung der Finanzierungslasten und letztlich die Rationalität und Objektivität des EU-Eigenmittelsystems.

Auch die Ausgabenprioritäten des EU-Budgets werden vorrangig unter dem Gesichtspunkt ihrer Kompensationsfunktion vereinbart. Aus dem Blickwinkel der Nettosaldo-Logik wird eine Haushaltsposition in der Finanziellen Vorausschau nicht mehr danach bewertet, ob eine gemeinsame europäische Politik einen europäischen Mehrwert erzielen kann. Stattdessen steht im Vordergrund, ob die Einzahlungen für diese europäische Politik den monetären Rückflüssen aus dem EU-Budget entsprechen. Jeder Mitgliedstaat setzt sich entsprechend dieser Logik in Politikbereichen, in denen er mit hohen Rückflussquoten rechnen kann, für eine Ausweitung des Budgetrahmens ein; überall dort, wo seine Rückflüsse wahrscheinlich unterdurchschnittlich sein werden, drängt er auf Einsparungen. Dies führt in der Konsequenz dazu, dass potenzielle Rückflussquoten wichtiger werden, als ein gemeinsam erkannter Handlungsbedarf. Das Verfahren führt so zu einer übermäßigen Verfestigung einmal vereinbarter Kompromisse und zur Erstarrung einmal eingeführter Regelungen.

27 Diese Sichtweise manifestiert sich in Szenarien und Modellen, die der Bewertung von Vorschlägen während der Finanzverhandlungen dienen sollen und sich vornehmlich auf die Auswirkungen für die nationale Nettosaldo-Position konzentrieren. So auch Friedrich Heinemann: EU-Finanzplanung 2007-2013. Haushaltsoptionen, Verteilungswirkungen und europäischer Mehrwert, in: Reihe ,Europa vordenken' der BertelsmannStiftung, Juni 2005.

28 Im Zuge der Beitrittsverhandlungen operationalisierte die Kommission das Konzept der Nettosalden und verwandte es erstmals auch, um ein eigenes politisch gewolltes Ziel zu begründen. Damit die neuen Mitgliedstaaten aus Mittel- und Osteuropa nicht im ersten Jahr ihrer Mitgliedschaft mehr Finanzmittel an den EU-Haushalt abführen mussten als sie in Form von Agrar- und Strukturhilfen zurück bekamen, errechnete die Kommission im Mai 2002 das voraussichtliche Nettosaldo für jedes Beitrittsland und sagte den potenziellen Nettozahlern Tschechien, Zypern, Malta und Slowenien eine entsprechend hohe Kompensationszahlung der EU zu. 
Auch in der Agenda 2007 hat der Europäische Rat viele Sonderregelungen, die explizit an besondere Bedingungen gekoppelt oder nur für einen befristeten Zeitraum vereinbart waren, einfach fortgeschrieben, wie zum Beispiel das Sonderförderprogramm zu Gunsten dünn besiedelter Regionen in Schweden, Finnland und Österreich oder die Sonderzahlung zur Unterstützung des nordirischen Friedensprozesses. Die Verfestigung der einmal eingeführten Sonderregelungen und die deutliche Ausweitung dieser nicht regelkonformen Leistungszusagen im vorliegenden Kompromiss birgt langfristig eine doppelte Gefahr:

- Die Komplexität und Intransparenz des EU-Finanzrahmens wird weiter zunehmen und die europäische Förderpolitik wird noch deutlicher als bisher nach fiskalischen Kriterien ausgehandelt.

- Der Missbrauch der EU-Förderprogramme zur Feinjustierung nationaler Nettosaldobilanzen bedeutet nicht nur eine Aufweichung der objektiven Förderkriterien, sondern auch eine Relativierung gemeinsamer europäischer Standards. Die Bezugsberechtigung wird zur politischen Verhandlungsmasse und läuft Gefahr, beliebig zu werden. Die Ausnahme droht zur Regel zu werden.

Jedoch benötigt das System als Konsequenz der Nettosaldo-Logik diese Vielzahl von ,Stellschrauben'; sie ermöglichen immer wieder das Schnüren neuer Kompromisspakete, ohne das Gesamtsystem selbst in Frage zu stellen. Die Verhandlungszwänge fordern nahezu den Missbrauch der europäischen Ausgabenpolitik zur Feinjustierung nationaler NettosaldoBilanzen heraus. Damit wird die Anpassungsfähigkeit der europäischen Politiken an veränderte Rahmenbedingungen erschwert. Die Neuausrichtung der EU-Ausgaben und die Verständigung auf neue Ausgabenziele wird fast unmöglich, denn jeder Mitgliedstaat bewertet neue Vorschläge, also Veränderungen des Status quo, selbstverständlich zunächst an den Auswirkungen auf den nationalen Nettosaldo. Die politischen Gestaltungsspielräume von und für europäische Politik werden so potentiell kleiner werden.

Eine mögliche negative integrationspolitische Langzeitfolge dieser Politik könnten darüber hinaus weiter sinkende Zustimmungsraten zur EU sein. Die für den politischen Konsens notwendige Komplexität und Intransparenz bedeutet für den Unionsbürger, dass sowohl die Finanzierungskosten der EU als auch die Legitimation der europäischen Ausgabenpolitik und die Verantwortlichkeiten für diese Politik unverständlich bleiben. Hingegen bleiben die Folgen der systemisch angelegten Ineffizienz, die regelmäßig in Presseberichten über unnötige Förderprogramme aus dem EU-Haushalt oder sinnlose Einzelprojekte dokumentiert werden, im Bewusstsein der nationalen Steuerzahler präsent. ${ }^{29}$

29 Vgl. „Brüssel im Regen“, in: Der Spiegel, Nr. 50/2005, S. 42-44. 